Study's endpoint was the proportion of patients achieving sustained complete remission of $\mathrm{BD}$ for at least 3 years after cessation of the anti-TNF agent.

Results: A total of 28 patients in whom infliximab and/or adalimumab treatment was given, always combined with azathioprine unless not tolerated $(n=2)$, and discontinued anytime before December 2013 were eligible for analysis. Following cessation of successful anti-TNF treatment (median duration of 2 years) $13 / 28$ patients achieved the study's end-point. The main reason for anti-TNF administration was sight-threatening ocular disease $(n=12)$ or intestinal disease $(n=1)$. The remaining 15 patients relapsed within 1.5 year (main reason for anti-TNF: ocular disease, $n=9$; neuro-BD, $n=2$; severe mucocutaneous disease, $n=3$; intestinal disease, $n=1$; median treatment duration of 24 months); $12 / 15$ were successfully re-treated with anti-TNF. So far, 3 of them (ocular disease, $n=2$; neuro-BD, $n=1$ ) have achieved the study's end-point (median re-treatment duration of 2 years). Overall, our 16 patients who achieved the study's end-point (57\%) are in complete disease remission ranging from 3 to 12 years (5.7 years, median). Nine patients with severe ocular disease are currently any drug-free (32\%), whereas the 7 remaining patients are on low doses of conventional immunosuppressive therapy $(25 \%)$. Notably, those patients on drug-free remission had shorter median disease duration at initiation of anti-TNF treatment, compared to the remaining patients (1 versus 3 years, respectively).

Conclusions: Sustained drug-free remission for many years after cessation of successful anti-TNF treatment is feasible in some patients with severe BD. Since anti-TNF-induced "cure" can never be differentiated from a spontaneous remission by natural history, further studies should examine whether early anti-TNF treatment must be intended for every patient with severe BD. References:

[1] Hatemi G, et al. EULAR recommendations for the management of Behçet disease. Ann Rheum Dis. 2008 Dec;67(12):1656-62.

Disclosure of Interest: None declared

DOI: 10.1136/annrheumdis-2017-eular.5338

\section{FRI0307 AN OUTCOME SURVEY OF 100 PATIENTS WITH CEREBRAL VENOUS SINUS THROMBOSIS DUE TO BEHÇET'S SYNDROME FOLLOWED UP AT A SINGLE, DEDICATED CENTER}

E.A. Kurt ${ }^{1}$, Y. Ozguler ${ }^{1}$, N. Kocer ${ }^{2}$, D. Ucar ${ }^{3}$, U. Uygunoglu ${ }^{4}$, C. Islak ${ }^{2}$, S. Ugurlu ${ }^{1}$, G. Hatemi ${ }^{1}$, S. Saip ${ }^{4}$, M. Melikoglu ${ }^{1}$, I. Fresko ${ }^{1}$, V. Hamuryudan ${ }^{1}$, Y. Ozyazgan ${ }^{3}$, S. Yurdakul ${ }^{1}$, A. Siva ${ }^{4}$, H. Yazici ${ }^{1}$, E. Seyahi ${ }^{1}{ }^{1}{ }^{1}$ Department of Internal Medicine Division of Rheumatology; ${ }^{2}$ Department of Radiology; ${ }^{3}$ Department of Ophthalmology; ${ }^{4}$ Department of Neurology, Istanbul University Cerrahpasa Medical Faculty, Istanbul, Turkey

Background: Behcet's syndrome (BS) is a well-recognized cause of cerebral venous sinus thrombosis (CVST).

Objectives: We aimed to assess the outcome of a large cohort of patients with CVST due to BS attending a single dedicated center.

Methods: We identified 100 ( $81 \mathrm{M} / 19$ F) patients with BS who were diagnosed as having CVST. All contacted were called back to the outpatient clinic for clinical and imaging re-evaluation.

Results: The mean age of the patients at the onset of the symptoms was $28 \pm 10$ years. A total of 48 patients developed CVST before or at the onset of ISG fulfillment, while 52 developed CVST after a median 3 [2-8] years of ISG fulfillment. Superior sagittal $(n=47)$ and transverse sinuses $(n=46)$ were most commonly involved followed by sigmoid sinus $(n=26)$ and jugular vein thrombosis $(n=15)$. A total of $59(53 \mathrm{M} / 6 \mathrm{~F})$ patients had vascular involvement in addition to CVST: these were deep vein thrombosis of the lower extremities $(n=47)$, pulmonary artery involvement $(n=17)$, Budd-Chiari syndrome $(n=9)$, vena cava superior thrombosis $(n=6)$ and major arterial disease $(n=3)$. In about half $(32 / 59)$, CVST preceded any type of additional vascular involvement. Eye involvement was seen in 37, parenchymal CNS involvement in 8 (all later than CVST) and gastrointestinal involvement in 5 patients.

Seven patients had died. By the end of the study, 87 patients were alive and contacted with a median follow-up time of 11 [6-15] years. Only 6 patients had a relapsing CVST course.

Information about medical treatment was present in detail in 87 patients of whom 75 received short courses of glucocorticoids with $(n=12)$ or without anti-coagulants. A total of 81 patients received immunosuppressive agents, most commonly azathioprine. Four patients underwent lumbo-peritoneal shunting surgery (1 was successful) and 1 with arterio-venous fistula underwent vascular embolization.

Fifty patients were re-evaluated at the clinic. None had of symptoms of intracranial hypertension. Ophthalmological examination showed that 17 patients had complications such as bilateral optic atrophy $(n=3)$, bilateral papilledema $(n=5)$, bilateral optic disc pallor $(n=4)$ and fibrotic scars around optic disc $(n=5)$. Sensorineural type hearing loss was detected in 4 patients. Neurological examination was found to be normal in 43 patients with isolated CSVT, whereas abnormal in the remaining 7 patients with concomitant parenchymal CNS involvement.

Cranial MR/MR venographies at the end of follow-up, were abnormal in 36 patients showing occlusion/ irregularity/ hypoplasia or collaterals in the sagittal $(n=19)$ or transverse sinus $(n=17)$. In the remaining 14 , MR venographies were normal.

Conclusions: CVST due to BS is closely associated with vascular involvement in the body and may be considered as a risk factor for future vascular involvement. CVST relapses are rare; however, the course is not uneventful: visual acuity or field may be impaired totally or partially because of optic disc atrophy; in addition hearing deficits may occur.

Disclosure of Interest: None declared

DOI: 10.1136/annrheumdis-2017-eular.6323

\section{FRI0308 PREDICTORS OF HYPOGAMMAGLOBULINAEMIA IN RITUXIMAB TREATED PATIENTS. A RETROSPECTIVE ANALYSIS ON A MONOCENTRIC COHORT}

R. Padoan, M. Felicetti, M. Gatto, P. Polito, F. Ometto, D. Astorri, L. Friso, F. Cozzi, A. Doria, L. Punzi, F. Schiavon. Operative Unit of Rheumatology, Department of Medicine DIMED, University of Padova, Padova, Italy

Background: Rituximab (RTX), a chimeric monoclonal antibody against CD20, is increasingly used in the treatment of B-cell lymphomas and autoimmune conditions. It has been shown that some patients develop hypogammaglobulinaemia after treatment.

Objectives: To assess frequency and predictors of hypogammaglobulinaemia after RTX treatment in a monocentric cohort of patients affected with granulomatosis with polyangiitis (GPA), microscopic polyangiits (MPA) and connective tissue diseases (CTD).

Methods: We retrospectively reviewed all patients receiving RTX and concomitant/sequential immunosuppressants between 2007 and 2016 in a single rheumatologic center. Serum levels of total Ig and lymphocyte subsets were recorded at the time of RTX administration and 3-6 months later. We investigated the frequency of hypogammaglobulinaemia $(\mathrm{lgG}<6 \mathrm{~g} / \mathrm{L})$ and its related events. Results: 72 patients, 30 (41.6\%) GPA/MPA, $25(34.7 \%)$ systemic lupus erythematosus, $13(18.1 \%)$ systemic sclerosis and $4(5.6 \%)$ poly-dermatomyositis were treated with RTX. We analyzed 113 RTX infusions, with 41 (36.2\%) retreatments (median 2 [2-6]). We excluded 12 patients/18 infusions due to incomplete data. RTX was administered at the dosage of $1000 \mathrm{mg}$ twice in $68.1 \%$ of patients and $375 \mathrm{mg} / \mathrm{m}^{2}$ weekly in $31.9 \%$. IgG levels after RTX infusion were available in $68(71.6 \%)$ patients. We observed a significant decrease of IgG levels between baseline and 3-6 months after RTX infusion in all patients $(0.001)$. The frequency of patients with $\lg G<6 \mathrm{~g} / \mathrm{L}$ was $22.1 \%$, and $8.8 \%$ had $\lg G<4 \mathrm{~g} / \mathrm{L}$, significantly higher in GPA/MPA patients (0.008), with short disease duration (0.001), lower IgG levels at baseline (0.008), higher prednisone equivalent (PDE) cumulative dosage per year (0.006) and higher daily PDE dosage after RTX (0.001) (Table 1). After RTX, all patients had complete B-cells depletion.

At univariate analysis, $\lg \mathrm{g}<6 \mathrm{~g} / \mathrm{L}$ was associated with GPA/MPA diagnosis $(0.006$, OR 6 [1.5-24.2]), disease duration (<0.001, OR $0.2[0.1-0.9]$ ), RTX $375 \mathrm{mg} / \mathrm{m}^{2}$ weekly protocol ( $\mathrm{p}=0.017$, OR 4.1 [1.2-13.9]), PDE cumulative dosage per year $(<0.001$, OR 6.6 [1.3-33.6]), daily PDE intake $>15 \mathrm{mg} /$ day after $\operatorname{RTX}(<0.001$, OR 12.7 [3.1-52.5]) and lgG levels before RTX $(<0.001$, OR 18 [1.8-178]).

At multivariate analysis, daily PDE intake after RTX ( $>15 \mathrm{mg} /$ day) and GPA/MPA diagnosis resulted independent predictive factors for hypogammaglobulinaemia $(p=0.03$, OR 9.5, [2.2-41.7] and $p=0.43$, OR 4.7, [1.1-21.5]).

Patients affected with GPA/MPA were compared to CTD, as reported in Table 2. GPA/MPA patients at infusion were older (0.002), presented shorter disease duration (0.001), lower IgG levels at baseline $(<0.001)$ despite lower rate of nephrotic syndrome $(0.003)$. Moreover they were treated more frequently with Azathioprine $(0.007)$, RTX $375 \mathrm{mg} / \mathrm{m}^{2}$ weekly protocol $(<0.001)$ and higher PDE cumulative and daily dosage $(<0.001,0.01$ respectively).

Only 5 patients $(5.2 \%)$ experienced severe infections within 12 months, more frequently in $\lg G<6 \mathrm{~g} / \mathrm{L}$ patients $(0.007)$.

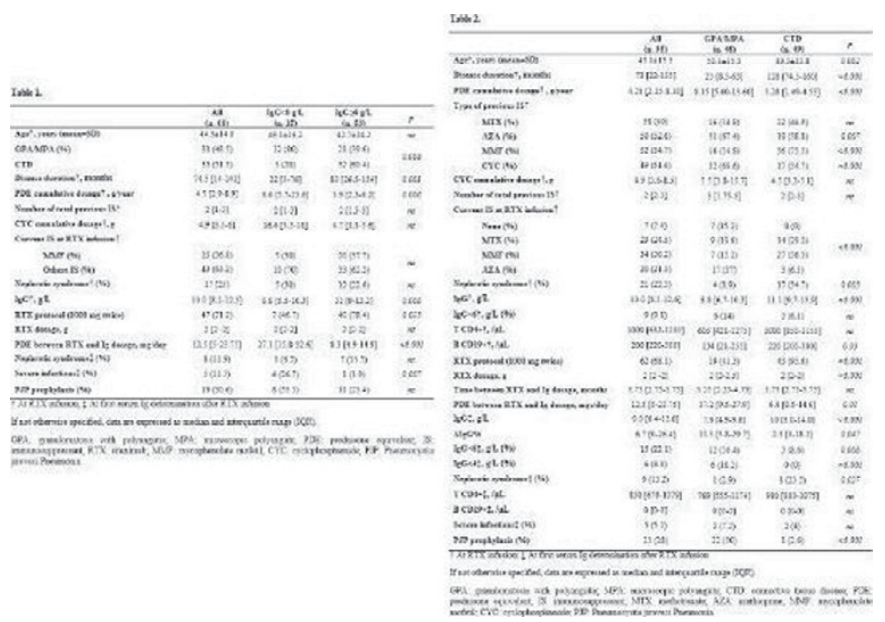

Conclusions: In autoimmune rheumatic diseases, diagnosis of GPA/MPA and glucocorticoid therapy resulted independent predictors of hypogammagobulinaemia after RTX treatment. Despite low IgG levels were associated with higher infections risk, rare severe infectious events were observed. 